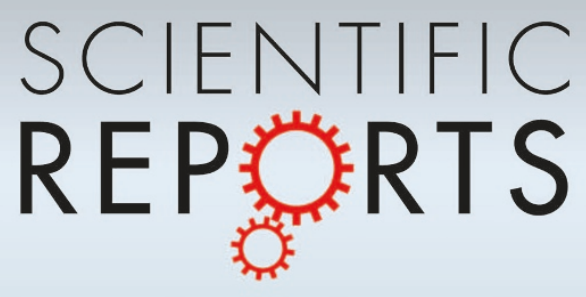

OPEN

SUBJECT AREAS:

APPLIED PHYSICS

MICROWAVE PHOTONICS

\author{
Received \\ 17 February 2014 \\ Accepted \\ 7 May 2014 \\ Published \\ 9 June 2014
}

Correspondence and requests for materials should be addressed to H.S.C. (hansomchen@

zju.edu.cn) or B.L.Z.

(blzhang@ntu.edu.sg)

\section{Realization of deep subwavelength resolution with singular media}

\author{
Su $X u^{1,2,3,4}$, Yuyu Jiang ${ }^{1,2}$, Hongyi $X u^{3,4}$, Junxia Wang ${ }^{1,2}$, Shisheng Lin ${ }^{1,2}$, Hongsheng Chen ${ }^{1,2}$ \\ \& Baile Zhang ${ }^{3,4}$
}

\begin{abstract}
'The Electromagnetics Academy at Zhejiang University, Zhejiang University, Hangzhou 310027 , China, ${ }^{2}$ State Key Laboratory of Modern Optical Instrumentation, Zhejiang University, Hangzhou 310027, China, ${ }^{3}$ Division of Physics and Applied Physics, School of Physical and Mathematical Sciences, Nanyang Technological University, Singapore 637371, Singapore, ${ }^{4}$ Centre for Disruptive Photonic Technologies, Nanyang Technological University, Singapore 637371, Singapore.
\end{abstract}

The record of imaging resolution has kept being refreshed in the past decades and the best resolution of hyperlenses and superlenses so far is about one out of tens in terms of wavelength. In this paper, by adopting a hybrid concept of transformation optics and singular media, we report a broadband meta-lens design methodology with ultra-high resolution. The meta-lens is made of subwavelength metal/air layers, which exhibit singular medium property over a broad band. As a proof of concept, the subwavelength imaging ability is demonstrated over a broad frequency band from $1.5-10 \mathrm{GHz}$ with the resolution varying from $1 / 117$ to $1 / 17$ wavelength experimentally.

$\mathrm{T}$ he resolution of conventional imaging systems is fundamentally limited to the diffraction limit ${ }^{1}$ because evanescent waves, which carry subwavelength information, decay exponentially during propagation in free space. Serving as a powerful impetus to semiconductor fabrication, biological studies, material science and medicine development, the unconventional imaging techniques that can break the diffraction limit have attracted considerable interest in recent years. In addition to the near-field scanning optical microscope ${ }^{2,3}$ and stimulated emission depletion microscope $e^{4}$, perfect len $s^{5}$ and hyperlens ${ }^{6}$ perform the function to recover subwavelength details of objects to be imaged. By folding electromagnetic space, a perfect lens can achieve unlimited resolution in theory and has been demonstrated with left-handed metamaterials composed of artificial "atoms" in experiments ${ }^{7-14}$. A hyperlens is made of anisotropic media with a hyperbolic $k$ surface, in which evanescent waves can be converted to propagating ones. The best realized resolution of all fabricated hyperlenses so far is about one out of tens in terms of wavelength ${ }^{15-19}$. To refresh this record further has practical demand but is widely acknowledged to be challenging.

Different from perfect lens and hyperlens, canalization regimes ${ }^{20-26}$, which do not involve left-handed materials and amplification of evanescent waves, can realize subwavelength imaging as well. This strategy is based on the eigenmode analysis of photonic crystals. The media for subwavelength imaging are constructed by metallic rod array, whose effective parameters are defined with a nonlocal effective medium model ${ }^{27-31}$. Several proofs of concept have been experimentally demonstrated in microwave regimes ${ }^{32-35}$ and exhibit potential applications in near-field microscopy and in medical imaging from microwave frequencies to infrared frequencies ${ }^{36}$.

Recently, singular media ${ }^{37,38}$ have shown potential ability in realizing deep subwavelength imaging. Singular media have the property that some components of the permittivity or permeability tensors are infinite or almost zero and therefore have already been taken into consideration of various potential electromagnetic applications, e.g. waveguide ${ }^{39}$, optical isolation ${ }^{40}$, metamaterials with ultrahigh refractive index ${ }^{41-43}$, superlens ${ }^{44}$ and subwavelength channeling applications ${ }^{45-47}$.

In this Letter, with the use of transformation optics ${ }^{48-51}$ and singular media, we report a practical meta-lens design methodology to realize ultra-high resolution in terms of wavelength for transverse magnetic (TM) polarized waves. The alternate metallic and dielectric layers, which form effective permittivity with extremely high anisotropy and can be treated as a kind of singular media, are adopted in our design and fabrication. The meta-lens is demonstrated experimentally over a broad frequency band from $1.5-10 \mathrm{GHz}$ with the resolution varying from $1 / 117$ to $1 / 17$ wavelength.

\section{Results}

The scheme of a TM cylindrical meta-lens is shown in Fig. 1(a). Two point sources are located closely at the inner boundary of the lens with subwavelength distance. For a specific material, the dispersion relation of electromag- 
(a)

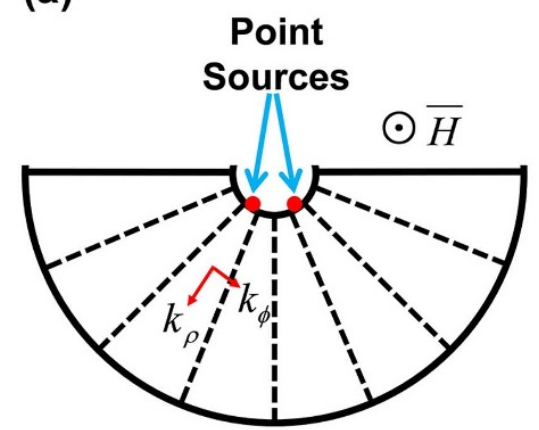

(c)

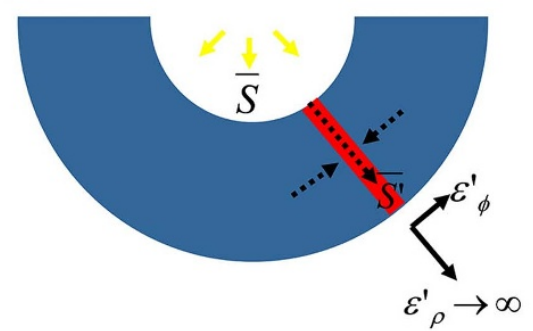

(b)

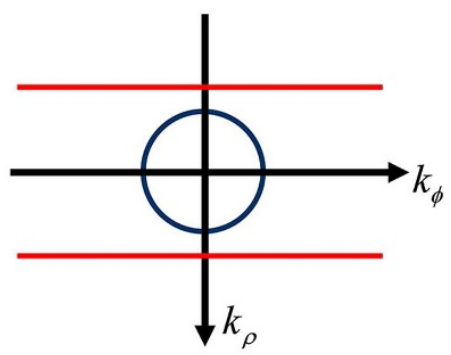

(d)

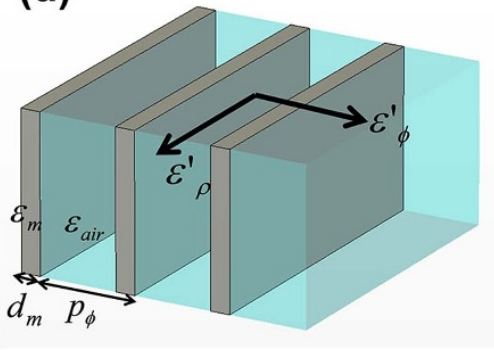

Figure $1 \mid$ (a) A two-dimensional scheme of an unconventional lens for TM waves. (b) The $k$ surfaces for an isotropic medium (circle in blue) and a singular medium (parallel lines in red). (c) The corresponding virtual space of materials in (b). In isotropic materials, energy flow $\bar{S}$ will spread out in all the directions. After squeezing the original virtual space (the regime in blue) in the azimuthal direction, $\overline{S^{\prime}}$ can only propagate in the transformed tube-like space (the regime in red) without spreading out. (d) The metamaterial with infinite anisotropy is made of metal-air structure with the period $p_{\phi}$ and the thickness of metal $d_{m}$.

netic waves can be written as $k_{\rho}^{2} / \varepsilon_{\phi}+k_{\phi}^{2} / \varepsilon_{\rho}=\omega^{2} \mu_{z}$ in cylindrical coordinates, where $k_{\rho}$ and $k_{\phi}$ are wave vectors along the $\rho$ and $\phi$ directions, $\varepsilon_{\phi}$ and $\varepsilon_{\rho}$ are the permittivities along the $\phi$ and $\rho$ directions, $\mu_{z}$ is the permeability along $z$ direction, and $\omega$ is the angular frequency of incident waves. The $k$ surface for free space exhibits a circular shape (blue curve in Fig. 1(b)) and the energy flow of electromagnetic waves $\bar{S}$ will spread out during propagation because of diffraction and loss of evanescent waves with tangential wave vector $k_{\phi}>k_{0}$. The medium with hyperbolic dispersion, which is adopted in hyperlens design, keeps the large tangential wave vector coefficient $k_{\phi}$ propagating without being evanescent.

Because hyperbolic dispersion metamaterials always involve negative value of constitutive parameters, hyperlens achieved with these hyperbolic dispersion metamaterials can only work in a narrow frequency band. However, without hyperbolic dispersion metamaterials, we can still design a meta-lens to achieve the goal of subwavelength resolution. To overcome the energy spreading associated with the diffraction in free space, squeezing the whole space in the $\phi$ direction is a practicable transformation procedure, i.e. $\rho^{\prime}=\rho, \phi^{\prime}=1 / N \phi$, and $z^{\prime}=z$, where $N$ is the squeezing constant. From the transformation optics, it is known that the permittivity coefficient along the radial direction becomes infinite as $N$ tends to infinite. Therefore, the medium in the transformed space has the property of singular media. As $\varepsilon_{\phi}^{\prime}$ is a finite value and $\varepsilon_{\rho}^{\prime} \rightarrow \infty$, the dispersion relation for an electromagnetic wave with electric field perpendicular to $\mathrm{z}$ axis becomes $k_{\rho}^{2} / \varepsilon_{\phi}^{\prime}=\omega^{2} \mu_{z}$ (parallel lines in red, Fig. 1(b)), which implies that the Poynting vector of incident wave is always along the $\rho$ direction and the propagation velocity is controlled by the permittivity $\varepsilon_{\phi}^{\prime}$ and permeability $\mu_{z}$. The energy is confined in the transformed space and cannot spread out as the space transforms from free space to an extremely thin tube-like space. Thus, the information carried by the tangential waves can be preserved and the diffraction limit can be overcome. The corresponding changes in virtual space between isotropic medium (e.g. air) and suppressed medium with infinite anisotropy are plotted in Fig. 1(c). Therefore, a practical high-resolution meta-lens in terms of wavelength can be realized by a singular medium, but without hyperbolic dispersion relation.

As shown schematically in Fig. 1(d), metamaterial realized by alternate metallic and air layers with subwavelength period $p_{\phi}$ is a potential choice to achieve extremely high anisotropy. With the effective medium theory, the perpendicular and parallel coefficients of the effective permittivity can be calculated as $\varepsilon_{\phi}^{\prime}=\varepsilon_{\perp}=$ $\left(\varepsilon_{m} \varepsilon_{\text {air }}\right) /\left(F \varepsilon_{\text {air }}+(1-F) \varepsilon_{m}\right)$ and $\varepsilon_{\rho}^{\prime}=\varepsilon_{\|}=F \varepsilon_{m}+(1-F) \varepsilon_{\text {air }}$, where $F$ $=d_{m} / p_{\phi}$ is the filling factor of the metal, $\varepsilon_{m}$ is the permittivity of metal, and $\varepsilon_{a i r}$ is the permittivity of air. For the case of microwave regime, infinite permittivity of metal $\varepsilon_{m}$ and finite filling factor $F$ introduces a singular medium property (i.e. $\varepsilon_{\phi}^{\prime}=\varepsilon_{\perp}=1 /(1-F)$ and $\left.\varepsilon_{\rho}^{\prime}=\varepsilon_{\|} \rightarrow \infty\right)$. For the case of higher frequency or optical regime, the permittivity of metal is not an infinite value due to the dispersion, but the permittivity ratio between the absolute value of metal and air still tends to a large value, especially in the infrared spectrum. Therefore, alternate metal-air layers can exhibit approximately singular medium property in optical regime as they exhibit in microwave regime. In such a medium, the wave can only propagate along the $\rho$ direction and no energy can be spread out.

As an example, we simulate TM polarized electromagnetic waves with the frequency of $100 \mathrm{THz}$ incident onto the homogeneous medium with high anisotropy $\left(\left|\operatorname{Re}\left(\varepsilon_{y} / \varepsilon_{x}\right)\right|>40\right.$, Fig. 2(a) and the effective medium constructed with gold-air layered structure (the filling factor of gold is 0.1 , Fig. 2(b)), respectively. The simulated result is obtained with the use of the commercial software Comsol Multiphysics. The permittivity of gold is given by $\varepsilon=1-\frac{\omega_{p}^{2}}{\omega\left(\omega+i \omega_{c}\right)}$ and for bulk gold, the plasma frequency $\omega_{p}=2 \pi \times 2.175 \times 10^{15} \mathrm{~s}^{-1}$ and the collision frequency $\omega_{c}=2 \pi \times 6.5 \times 10^{12} \mathrm{~s}^{-1} 52$. The incidence angle is 30 degree. Two media in Fig. 2(a) and 2(b) have the same 

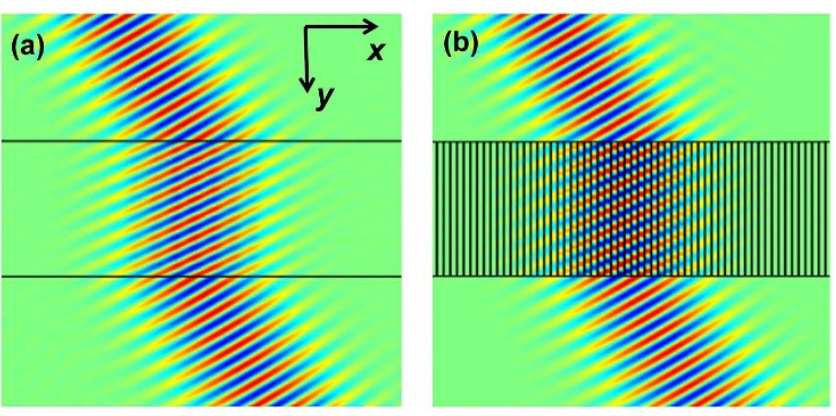

Figure $2 \mid$ Electromagnetic wave with the frequency of $100 \mathrm{THz}$ propagate through (a) the homogeneous medium with high anisotropy $\left(\left|\operatorname{Re}\left(\varepsilon_{y} / \varepsilon_{x}\right)\right|>40\right)$ and (b) the effective medium with the same effective parameters constructed with gold-air layered structure. The permittivity of gold is given by $\varepsilon=1-\frac{\omega_{p}^{2}}{\omega\left(\omega+i \omega_{c}\right)}$ and for bulk gold, the parameters are $\omega_{p}=2 \pi \times 2.175 \times 10^{15} s^{-1}$ and $\omega_{c}=2 \pi \times 6.5 \times 10^{12} s^{-1}$. The incidence angle is 30 degree and the filling factor of gold is 0.1 .

constitutive parameters based on the effective medium theory. From the results, one can see the field distributions are identical in these two cases, indicating the layered metal-air structure can be well characterized as a homogeneous singular medium. Following the Drude model, $\left|\operatorname{Re}\left(\varepsilon_{\text {gold }}\right)\right|$ will drop to a finite value from infinity as the frequency increases, especially when the frequency tends to the plasma frequency of gold. At this condition, the layered gold-air structure cannot exhibit the highly anisotropic property of finite $\varepsilon_{\perp}$ and infinite $\varepsilon_{\| \mid}$. This will introduce the loss of evanescent waves and therefore deteriorate the performance of hyperlens. However, this structure can still perform as a singular medium in a broad frequency band from microwave to Terahertz regimes as long as it meets the effective medium theory.

Fig. 3 shows the schematic of microwave meta-lens we realized. In order to simplify the fabrication, we separate the semicircle of the meta-lens into 18 tubes by 0.03 mm-thick Cleanwrap ${ }^{\circledR}$ aluminum foils and therefore the azimuthal periodicity of the tube is 10 degree. The metal-air structures are fixed by two pieces of synthetic glasses, whose radius of inner semicircle and of outer semicircle are $r=$ $1.5 \mathrm{~mm}$ and $R=500 \mathrm{~mm}$ respectively. The height of the meta-lens in $z$ direction is $h=200 \mathrm{~mm}$. Two slits, with the width of $0.3 \mathrm{~mm}$ and height of $200 \mathrm{~mm}$ on a horn antenna covered by an aluminum foil, are used as two separated point sources in the experiment. The distance between two slits is $1.7 \mathrm{~mm}$. In the experiment, a composite source, which is subwavelength, is connected to the meta-lens with contact terminals. According to the calculation above, the effective permittivities close to the inner semicircle are $\varepsilon_{\phi}=\varepsilon_{\perp}=1.1$ and $\varepsilon_{\rho}=$ $\varepsilon_{\|} \rightarrow \infty$, which match the requirement of meta-lens.
Both simulations and experiments are carried out in microwave regime to verify our idea. For the case of free space (Fig. 4(a)), the field distribution of two point sources with the distance $1.7 \mathrm{~mm}$ is identical to the one of a single point source because the distance between two point sources is far less than half of free space wavelength at $1.5 \mathrm{GHz}$. However, for the case of sources with our metalens, the TM waves excited by the same pair of point sources can be efficiently separated (Fig. 4(b)). In the experiment, the distance between the receiver and the center is $0.6 \mathrm{~m}$. The scanning angle $\phi$ varies from 0 to 180 degree. The experimental results at $1.5 \mathrm{GHz}$ agree well with simulated results (Fig. 4(c)). Moreover, in order to verify the broadband property of our meta-lens design, we measured the field at different frequencies. Fig. $4(\mathrm{~d}-\mathrm{f})$ are the experimental results at $10 \mathrm{GHz}$, from which one can see that the two points can also be efficiently separated in the imaging plane, indicating that our meta-lens can overcome the diffraction limit from $1.5 \mathrm{GHz}$ to $10 \mathrm{GHz}$ with the resolution varying from $1 / 117$ to $1 / 17$ wavelength.

\section{Discussion}

There are two limitations relating to the bandwidth and resolution of our design. The first one is the effective medium theory (EMT), which can be used only when the periodicity of the layered structure has to be much smaller than the wavelength of interest (e.g. smaller than $1 / 5$ wavelength). In our design, the periodicity of the layered Alfoam structure is $0.26 \mathrm{~mm}$, which corresponds to $1 / 5$ wavelength of $230 \mathrm{GHz}$. The other one is the diffraction limit, which means that the distance between the two imaging point at the outer boundary should be larger than $1 / 2$ wavelength. In the design, this distance is $500 \mathrm{~mm}$, which corresponds to $1 / 2$ wavelength of $0.3 \mathrm{GHz}$. Therefore we estimate that the bandwidth limitation of our design is $0.3-230 \mathrm{GHz}$.

One may question that the squeezing constant must be a definite number for a real practical sample. As a result, the radial component of permittivity cannot be infinite in experiment. However, even with a definite squeezing number, the effective radial permittivity still tends to infinite, as the permittivity of metal is infinitely large at microwave regime. Hence, a definite squeezing constant would not deteriorate the subwavelength imaging performance in our practical design. For higher frequency, e.g. optical regime, a definite squeezing parameter will deteriorate the imaging performance because it is difficult to find a kind of material whose permittivity tends to infinite, but a medium with highly anisotropic permittivity can be still achieved for subwavelength imaging with the use of layered structure in Fig. 2.

In conclusion, we introduce a meta-lens design methodology with deep subwavelength resolution combining transformation optics and singular media. We implement this concept and demonstrate a practical broadband meta-lens in microwave regime. The metalens is made of alternate metallic and air layers, which can be treated as a singular medium. The advantages of this design include ultrahigh-resolution (up to $\lambda / 100$ resolution at $1.5 \mathrm{GHz}$ in the experi-

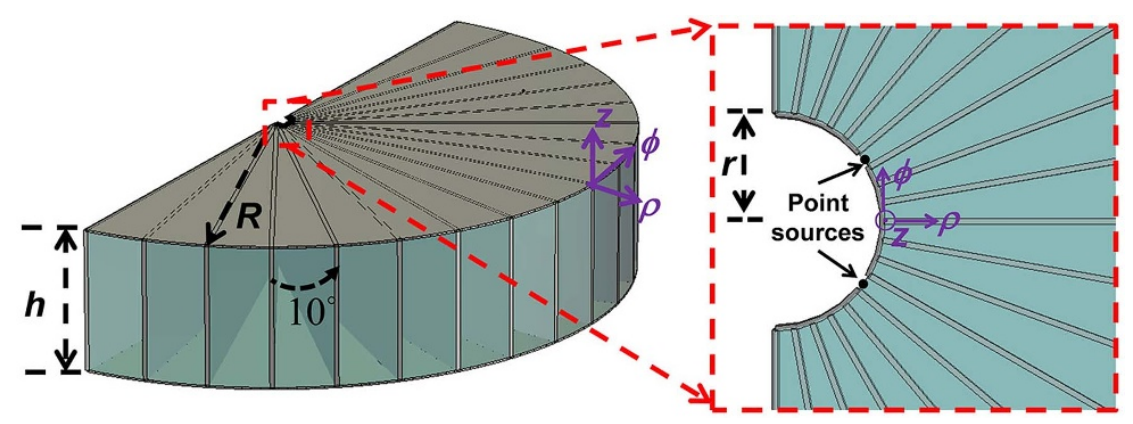

Figure $3 \mid$ The effective 3D scheme of our practical meta-lens for microwave. The inner and outer radius are $r=1.5 \mathrm{~mm}$ and $R=500 \mathrm{~mm}$ respectively. The height of meta-lens in $z$ direction is $h=200 \mathrm{~mm}$ and the azimuthal periodicity of the tube divided by 0.03 mm-thick Cleanwrap ${ }^{\circledR}$ aluminum foils is 10 degree. 

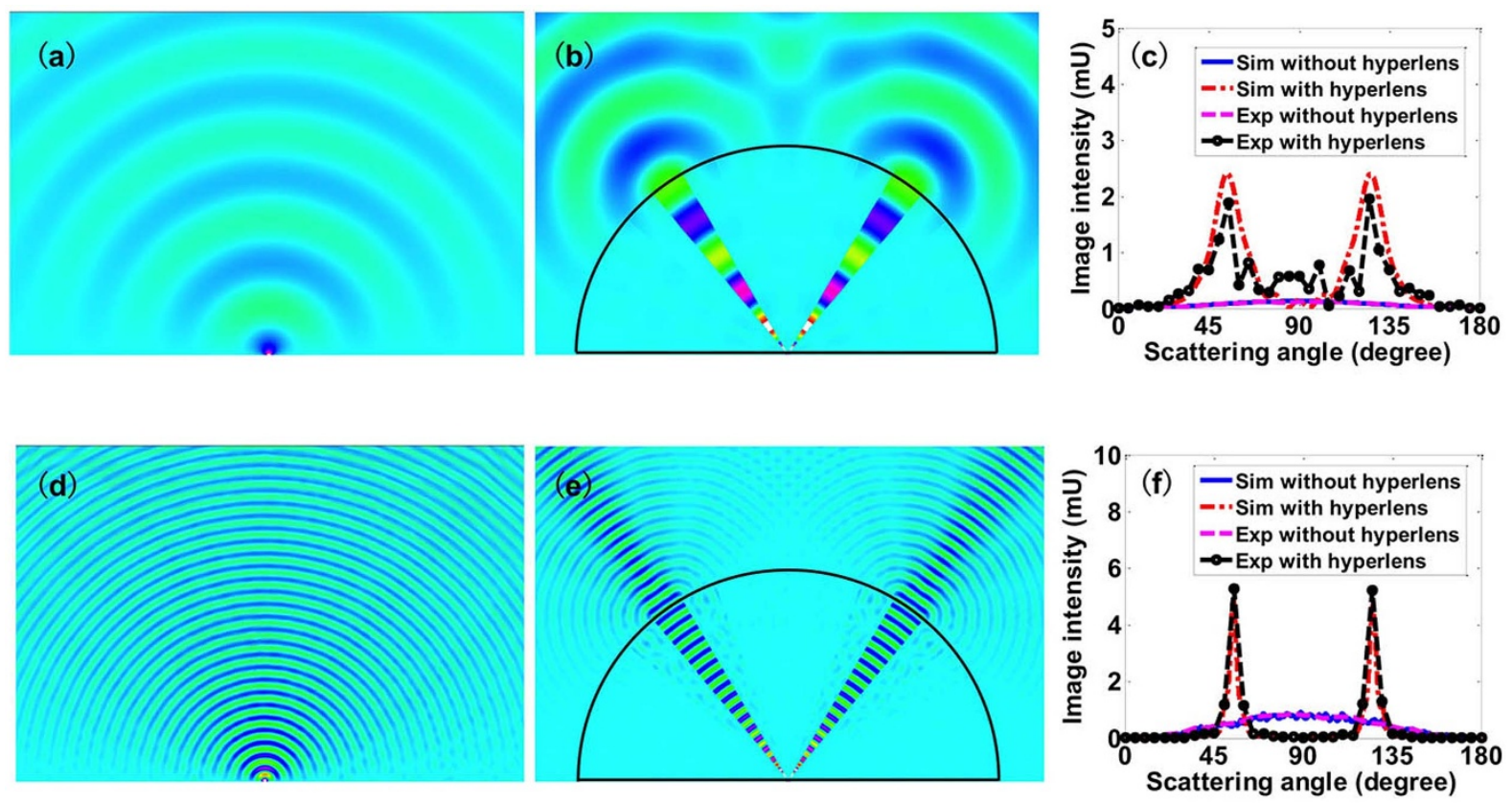

Figure $4 \mid$ The results at $1.5 \mathrm{GHz}$ are enclosed in the top panel $(\mathrm{a}-\mathrm{c})$ while the results at $10 \mathrm{GHz}$ are enclosed in the bottom panel (d-f): the simulated field distributions ( $a, d)$ for the case without meta-lens and $(b, e)$ for the case with meta-lens, and ( $c, f)$ the image intensity normalized by the input intensity. In the experiment, the distance between the receiver and the center is $0.6 \mathrm{~m}$.

ment) and broadband performance from $1.5-10 \mathrm{GHz}$ with the resolution varying from $1 / 117$ to $1 / 17$ wavelength. Our work provides an alternative way for future practical implementation of subwavelength imaging devices.

1. Abbe, E. Beiträge zur Theorie des Mikroskops und der mikroskopischen Wahrnehmung. Arch. Mikroskop. Anat. 9, 413-418 (1873).

2. Synge, E. H. A suggested method for extending microscopic resolution into the ultra-microscopic region. Philos Mag. 6, 356-362 (1928).

3. Betzig, E., Trautman, J. K., Harris, T. D., Weiner, J. S. \& Kostelak, R. L. Breaking the diffraction barrier: optical microscopy on a nanometric scale. Science 251, 1468-1470 (1991).

4. Gustafsson, M. G. L. Nonlinear structured-illumination microscopy: wide-field fluorescence imaging with theoretically unlimited resolution. Proc. Natl. Acad. Sci. 102, 13081-13086 (2005).

5. Pendry, J. B. Negative refraction makes a perfect lens. Phys. Rev. Lett. 85, 3966 (2000).

6. Jacob, Z., Alekseyev, L. V. \& Narimanov, E. Optical hyperlens: far-field imaging beyond the diffraction limit. Opt. Express 14, 8247-8256 (2006).

7. Zhang, X. \& Liu, Z. W. Superlenses to overcome the diffraction limit. Nat. Mater. 7, 435-441 (2008).

8. Liu, Z. W., Fang, N., Yen, T.-J. \& Zhang, X. Rapid growth of evanescent wave with a silver superlens. Appl. Phys. Lett. 83, 5184 (2003).

9. Grbic, A. \& Elefheriades, G. V. Overcoming the diffraction limit with a planar lefthanded transmission-line lens. Phys. Rev. Lett. 92, 117403 (2004).

10. Fang, N., Lee, H., Sun, C. \& Zhang, X. Sub-diffraction-limited optical imaging with a silver superlens. Science 308, 534-537 (2005).

11. Taubner, T., Korobkin, D., Urzhumov, Y., Shvets, G. \& Hillenbrand, R. Near-field microscopy through a SiC superlens. Science 313, 1595 (2006).

12. Durant, S., Liu, Z. W., Steele, J. M. \& Zhang, X. Theory of the transmission properties of an optical far-field superlens for imaging beyond the diffraction limit. J. Opt. Soc. Am. B 23, 2383-2392 (2006).

13. Liu, Z. W. et al. Far-field optical superlens. Nano Lett. 7, 403-408 (2007).

14. Liu, Z. W. et al. Experimental studies of far-field superlens for sub-diffractional optical imaging. Opt. Express 15, 6947-6954 (2007).

15. Salandrino, A. \& Engheta, N. Far-field subdiffraction optical microscopy using metamaterial crystals: theory and simulations. Phys. Rev. B 74, 075103 (2006).

16. Lee, H., Liu, Z. W., Xiong, Y., Sun, C. \& Zhang, X. Development of optical hyperlens for imaging below the diffraction limit. Opt. Express 15, 15886-15891 (2007).

17. Rho, J. et al. Spherical hyperlens for two-dimensional sub-diffractional imaging at visible frequencies. Nat. Commun. 1, 143 (2010).

18. Smith, E. J., Liu, Z., Mei, Y. F. \& Schmidt, O. G. System investigation of a rolledup metamaterial optical hyperlens structure. Appl. Phys. Lett. 95, 083104 (2009).
19. Wang, J., Xu, Y., Chen, H. \& Zhang, B. Ultraviolet dielectric hyperlens with layered grapheme and boron nitride. J. Mater. Chem. 22, 15863-15868 (2012).

20. Belov, P. A., Simovski, C. R. \& Ikonen, P. Canalization of subwavelength images by electromagnetic crystals. Phys. Rev. B 71, 193105 (2005).

21. Belov, P. A. \& Hao, Y. Subwavelength imaging at optical frequencies using a transmission device formed by a periodic layered metal-dielectric structure operating in the canalization regime. Phys. Rev. B 73, 113110 (2006).

22. Li, X., He, S. \& Jin, Y. Subwavelength focusing with a multilayered Fabry-Perot structure at optical frequencies. Phys. Rev. B 75, 045103 (2007).

23. Shvets, G., Trendafilov, S., Pendry, J. B. \& Sarychev, A. Guiding, focusing, and Sensing on the subwavelength scale using metallic wire arrays. Phys. Rev. Lett. 99, 053903 (2007).

24. Ikonen, P., Simovski, C., Tretyakov, S., Belov, P. \& Hao, Y. Magnification of subwavelength field distributions at microwave frequencies using a wire medium slab operating in the canalization regime. Appl. Phys. Lett. 91, 104102 (2007).

25. Silveirinha, M. G., Belov, P. A. \& Simovski, C. R. Ultimate limit of resolution of subwavelength imaging devices formed by metallic rods. Opt. Lett. 33, 1726-1728 (2008).

26. Kotyński, R. \& Stefaniuk, T. Comparison of imaging with sub-wavelength resolution in the canalization and resonant tunneling regimes. J. Opt. A: Pure Appl. Opt. 11, 015001 (2009).

27. Belov, P. A. et al. Strong spatial dispersion in wire media in the very large wavelength limit. Phys. Rev. B 67, 113103 (2003).

28. Silverinha, M. G., Alu, A. \& Engheta, N. Parallel-plate metamaterials for cloaking structures. Phys. Rev. E 75, 036603 (2007).

29. Orlov, A. A., Voroshilov, P. M., Belov, P. A. \& Kivshar, Y. S. Engineered optical nonlocality in nanostructured metamaterials. Phys. Rev. B 84, 045424 (2011).

30. Chebykin, A. V. et al. Nonlocal effective medium model for multilayered metaldielectric metamaterials. Phys. Rev. B 84, 115438 (2011).

31. Chebykin, A. V., Orlov, A. A., Simovski, C. R., Kivshar, Y. S. \& Belov, P. A. Nonlocal effective parameters of multilayered metal-dielectric metamaterials. Phys. Rev. B 86, 115420 (2012).

32. Belov, P. A., Hao, Y. \& Sudhakaran, S. Subwavelength microwave imaging using an array of parallel conducting wires as a lens. Phys. Rev. B 73, 033108 (2006).

33. Belov, P. A. et al. Transmission of images with subwavelength resolution to distances of several wavelengths in the microwave range. Phys. Rev. B 77, 193108 (2008).

34. Zhao, Y. et al. Magnification of subwavelength field distributions using a tapered array of metallic wires with planar interfaces and an embedded dielectric phase compensator. New J. Phys. 12, 103045 (2010).

35. Belov, P. A. et al. Experimental demonstration of multiwire endoscopes capable of manipulating near-fields with subwavelength resolution. Appl. Phys. Lett. 97, 191905 (2010).

36. Simovski, C. R., Belov, P. A., Atrashchenko, A. V. \& Kivshar, Y. S. Wire metamaterials: physics and applications. Adv. Mater. 24, 4229-4248 (2012). 
37. Smith, D. R. \& Schurig, D. Electromagnetic wave propagation in media with indefinite permittivity and permeability tensors. Phys. Rev. Lett. 90, 077405 (2003).

38. Sihvola, A., Tretyakov, S. \& de Baas, A. Metamaterials with extreme material parameters. J. Commun. Technol. Electron. 52, 986 (2007).

39. Edward, B., Alu, A., Young, M. E., Silveirinha, M. \& Engheta, N. Experimental verification of epsilon-near-zero metamaterial coupling and energy squeezing using a microwave waveguide. Phys. Rev. Lett. 100, 033903 (2008).

40. Davoyan, A. R., Mahmoud, A. M. \& Engheta, N. Optical isolation with epsilonnear-zero metamaterials. Opt. Express 21, 3279-3286 (2013).

41. Shen, J. T., Catrysse, P. B. \& Fan, S. H. Mechanism for designing metallic metamaterials with a high index of refraction. Phys. Rev. Lett. 94, 197401 (2005).

42. Shin, J., Shen, J. T. \& Fan, S. H. Three-dimensional metamaterials with an ultrahigh effective refractive index over a broad bandwidth. Phys. Rev. Lett. 102, 093903 (2009).

43. Choi, M. et al. A terahertz metamaterial with unnaturally high refractive index. Nature 470, 369-373 (2011).

44. Silverinha, M. G., Fernandes, C. A. \& Costa, J. R. Superlens made of a metamaterial with extreme effective parameters. Phys. Rev. B 78, 195121 (2008).

45. Catrysse, P. B. \& Fan, S. H. Routing for deep-subwavelength optical beams and images without reflection and diffraction using infinitely anisotropic metamaterials. Adv. Mater. 25, 194-198 (2012).

46. Silveirinha, M. \& Engheta, N. Tunneling of electromagnetic energy through subwavelength channels and bends using epsilon-near-zero materials. Phys. Rev. Lett. 97, 157403 (2006).

47. Ishii, S., Kildishev, A. V., Narimanov, E., Shalaev, V. \& Drachev, V. P. Subwavelength interference pattern from volume plasmon polaritons in a hyperbolic medium. Laser \& Photon. Rev. 7, 265-271 (2013).

48. Pendry, J. B., Schurig, D. \& Smith, D. R. Controlling electromagnetic fields. Science 312, 1780-1782 (2006).

49. Leonhardt, U. Optical conformal mapping. Science 312, 1777-1780 (2006).

50. Yan, M., Yan, W. \& Qiu, M. Cylindrical superlens by a coordinate transformation. Phys. Rev. B 78, 125113 (2008).

51. Tseng, M. \& Psaltis, D. Magnifying perfect lens and superlens design by coordinate transformation. Phys. Rev. B 77, 035122 (2008).
52. Linden, S. et al. Magnetic response of metamaterials at 100 terahertz. Science 306, 1351-1353 (2004).

\section{Acknowledgments}

This work was sponsored by the National Natural Science Foundation of China under Grants Nos. 61322501, 61275183, and 60990322, the National Program for Special Support of Top-Notch Young Professionals, the Foundation for the Author of National Excellent Doctoral Dissertation of PR China under Grant No. 200950, the Program for New Century Excellent Talents (NCET-12-0489) in University, the K. P. Chao's High Technology Development Foundation, the Fundamental Research Funds for the Central Universities, Nanyang Technological University under Start-Up Grants, Singapore Ministry of Education under Grant No. Tier 1 RG27/12 and Grant No. MOE2011-T3-1-005.

\section{Author contributions}

B.Z. conceived the original idea of the study. S.X., H.X. and J.W. performed the analysis and numerical simulations. S.X. and Y.J. carried out the experiment. H.C., S.L. and B.Z. interpreted the results and supervised the project. S.X., B.Z. and H.C. wrote the manuscript. All authors reviewed the manuscript.

\section{Additional information}

Competing financial interests: The authors declare no competing financial interests.

How to cite this article: $\mathrm{Xu}, \mathrm{S}$. et al. Realization of deep subwavelength resolution with singular media. Sci. Rep. 4, 5212; DOI:10.1038/srep05212 (2014).

This work is licensed under a Creative Commons Attribution-NonCommercialNoDerivs 3.0 Unported License. The images in this article are included in the article's Creative Commons license, unless indicated otherwise in the image credit; if the image is not included under the Creative Commons license, users will need to obtain permission from the license holder in order to reproduce the image. To view a copy of this license, visit http://creativecommons.org/licenses/by-nc-nd/3.0/ 